\title{
El papel de las bibliotecas en el acceso a recursos web de calidad
}

\section{Andrés Fernández-Ramos}

\begin{abstract}
Documentalista, Agencia de Evaluación de Tecnologías Sanitárias. Instituto de Salud Carlos III, Madrid Profesor asociado, Dpto. de Biblioteconomía y Documentación Universidad Complutense de Madrid
\end{abstract}

Internet ha supuesto una drástica transformación en las formas de comunicación y de acceso a la información. La ingente cantidad de información disponible en la web y el hecho de que esta no pase por ningún filtro suponen un problema a la hora de localizar información de calidad. Las deficiencias de los buscadores y la falta de competencia informacional dificultan un acceso eficaz a los recursos valiosos existentes en la web. En este trabajo se describe el papel que la biblioteca juega en el acceso a la información de calidad en internet a través de las principales actuaciones que pueden llevar a cabo: la formación de usuarios en competencias informacionales relacionadas con la búsqueda y evaluación de información $y$ el desarrollo de herramientas y servicios que permitan el acceso a recursos de calidad disponibles en internet, como son las guías temáticas, los directorios temáticos o la inclusión de estos recursos en el catálogo.

Palabras clave: Recursos web; Bibliotecas; Alfabetización informacional; Directorios temáticos; Guías temáticas.

\section{Access high quality web resources: the determinant role of libraries}

Internet has dramatically changed the ways of communication and access to information. The large amount of information available on the web and the lack of filters for publication imply that people face great difficulties in accessing to high quality information. Deficiencies of Internet search engines and the lack of information literacy bring more restrictions to achieve an efficient access to valuable web resources. This paper 
aims to describe the key role of libraries in improving an efficienct access to high quality web resources: on the one hand, teaching information competencies related to searching and evaluating information, and on the other hand, developing services and tools, such as thematic guides, subject gateways, and inclusion of this kind of resources in the library catalogue.

Keywords: Web resources; Libraries; Information literacy; Subject gateways; Subject guides.

Recebido em 22.05.2013Aceito em 17.09.2013

\section{Introducción}

Internet, la más importante plasmación del desarrollo de las telecomunicaciones en la sociedad, y especialmente la World Wide Web, su parte más visible, han favorecido una drástica transformación en las formas de comunicación y de acceso a la información. Tradicionalmente las bibliotecas o centros de documentación han sido los principales puntos de acceso donde consultar las fuentes de información, por lo general en formato impreso. Sin embargo, hoy en día, a través de un ordenador o de un dispositivo móvil conectado a la red, cualquiera puede acceder a una ingente cantidad de contenidos, de diversas formas y características y de muy diversa procedencia.

En este nuevo entorno de acceso a la información, el gran problema con el que se encuentra el usuario es que no es capaz de abarcar y controlar todo lo que hay en la red, no sólo por la cantidad de recursos existentes, sino también por su heterogénea naturaleza. Esta sobreabundancia de información, que en principio podría parecer la panacea de la democratización del acceso a la información, supone un problema por la dificultad que entraña localizar lo que se busca. El volumen de información disponible en internet crece exponencialmente y además cualquiera puede colgar información en la web sin pasar por ningún filtro que asegure cierta calidad a lo que se publica, lo que supone que, junto con información de alta calidad, en internet existan una gran cantidad de recursos inservibles, irrelevantes o desdeñables.

Las herramientas más utilizadas para buscar información en internet son los motores de búsqueda, siendo Google el más utilizado de ellos (PINGDOM, 2013). Estos buscadores cuentan con programas informáticos de gran potencia para rastrear la web, compilar recursos e incorporarlos a sus bases de datos de forma automatizada. Sin embargo, presentan una serie de limitaciones a la hora de localizar información de calidad que pueden redundar en una considerable pérdida de tiempo y en la insatisfacción de las demandas de información. Muchos de estos problemas ya fueron planteados por Oppenheim et al. (2000), que señalaban las limitaciones de los motores de búsqueda en la presencia de 
registros duplicados, recuperación de documentos poco relevantes, recuperación de enlaces a páginas web que ya no existen o que han cambiado de url, el spamming, la ordenación inadecuada de los recurso recuperados, etc. Para estos autores el tamaño, heterogeneidad e inconsistencia de la web son un serio problema que implica que las respuestas de los motores de búsqueda no estén ligadas, generalmente, a una efectiva recuperación de información (MARTÍNEZ; RODRÍGUEZ, 2003). Por su parte, Codina y Abadal (2013), señalan los siguientes puntos débiles de los buscadores y de la web en general a nivel tecnológico: barreras en la indización, debido a la web invisible y a los errores y malas prácticas en la codificación; limitaciones en el cálculo de la relevancia, propias de cualquier sistema que calcule la relevancia de forma automática; limitaciones en la página de resultados; y limitaciones en el lenguaje de búsqueda.

Siendo esta la situación, nos encontramos ante un importante desafío tecnológico en el mundo de la información, que ha dado lugar a dos temas emergentes con los que los profesionales de la información vamos a tener mucho que ver (KOHL, 2009): preparar la información digital para que pueda ser correcta y fácilmente recuperada; y desarrollar la habilidad para identificar la información útil y de calidad que puede ser valiosa para los usuarios de las bibliotecas.

Las bibliotecas y centros de documentación, los grandes intermediarios entre la información y los usuarios, han desarrollado a lo largo de la historia instrumentos y herramientas para solventar, o al menos aliviar, el problema del acceso a grandes cantidades de recursos. Mediante la selección de los fondos que van a integrar su colección establecen un filtro de calidad de los mismos y mediante la organización, clasificación y descripción de los documentos, así como con los servicios que prestan y las herramientas que desarrollan, ayudan a los usuarios a localizar los recursos que satisfagan sus necesidades de información. Aunque tradicionalmente sus fondos estaban en soporte papel, desde hace tiempo los recursos electrónicos se han incorporado a la colección de las bibliotecas y también han pasado por los procesos de evaluación, selección, organización y descripción. Las colecciones bibliotecarias ya no están formadas exclusivamente por documentos en soportes tangibles que se adquieren por los métodos tradicionales, sino que también pueden formar parte de ellas documentos de acceso remoto que no son necesariamente propiedad de la biblioteca, como pueden serlo los recursos disponibles en internet (ESTIVILL; ABADAL, 2000). Estos documentos no dejan de ser recursos informativos $y$, por tanto, susceptibles de ser seleccionados y tratados según los procedimientos bibliotecarios correspondientes para ponerlos a disposición de los usuarios. La información científica disponible en internet es de gran importancia y las bibliotecas deben evaluar la calidad de esos recursos de cara a su incorporación a la colección. Por tanto, las bibliotecas tienen la responsabilidad ética y profesional de ofrecer la mejor información a sus 
usuarios, independientemente de que esta tenga un formato $u$ otro (HERRING, 2011).

Aunque la inclusión de este tipo de recursos en las bibliotecas presenta una serie de inconvenientes, como puede ser la falta de información necesaria para juzgar su credibilidad o los problemas de permanencia (cambios en la url, desaparición, etc.), la comunidad bibliotecaria es consciente de que es necesaria su incorporación. Así se puso de manifiesto en el estudio Delphi que realizó Cross (2002) en Estados Unidos entre directores de bibliotecas y personal del servicio de referencia, en el que se señaló que la solución a estos problemas pasa necesariamente por la formación específica y la colaboración entre centros.

No obstante, con la aparición de internet las formas de búsqueda y el acceso a la información han cambiado significativamente y la labor de intermediación de las bibliotecas ha disminuido. Las personas pueden acceder a una ingente cantidad de información en la red y los motores de búsqueda, cada vez más potentes y fáciles de manejar, les brindan la oportunidad de buscar la información por su cuenta sin necesidad de utilizar los catálogos, bases de datos y otras herramientas y servicios que las bibliotecas también ponen a su alcance. Sin embargo, el acceso a la información de calidad en internet presenta una serie de dificultades nada desdeñables, relacionadas en su mayoría con el hecho de que cualquiera puede publicar en la red sin pasar ningún tipo de filtro y que no siempre es fácil encontrar lo que se busca y discriminar la información de calidad de la que no la tiene. La información en internet, aunque en sí misma no tiene por qué ser menos válida, no pasa por ningún tipo de filtro profesional (bibliotecarios, editores...) y eso supone que sea mucho más fácil encontrar información de baja calidad (LUCASSEN, SCHRAAGEN, 2011, p. 1232).

Es por este motivo que estas dos realidades, internet y las bibliotecas, se entrecruzan actualmente en un momento en que se necesitan mutuamente: por una parte la web necesita de las labores de los profesionales de la información para filtrar la información de calidad y hacerse accesible a los usuarios y, por otra, las bibliotecas y centros de documentación deben adaptarse a los nuevos soportes y formatos de la información en internet y facilitar el acceso a los usuarios a los numerosos recursos de calidad disponibles en la web. Como indica Codina (2000), a medida que internet vaya creciendo, será más y más necesario para los profesionales de la documentación disponer de criterios sólidos para saber evaluar recursos digitales y determinar su valor o su capacidad relativa para cumplir sus objetivos.

En este contexto, la aportación de las bibliotecas al acceso a información de calidad en internet es un reto y una obligación y puede articularse principalmente a través de dos tipos de actuaciones: por un lado mediante la formación a sus usuarios en las competencias informacionales relacionadas con la búsqueda y evaluación de información 
y, por otro, con el desarrollo de herramientas y servicios que permitan el acceso a recursos de calidad disponibles en internet.

\section{Formación en evaluación}

A pesar de que hoy en día el uso de internet para buscar información es lo más habitual, tanto en el ámbito académico como en el laboral o personal, existen numerosos estudios que indican una considerable falta de formación y de habilidades para buscar y evaluar eficazmente la calidad de la información en internet (MANDALIOS, 2013). Como señala muy acertadamente Breivick (1998, p. 23), refiriéndose al entorno académico, este problema no se soluciona por sí solo, es necesario dejar de asumir que la gente ya sabe buscar información o que fácilmente puede aprender a hacerlo sin ningún tipo de intervención; es por tanto imprescindible fomentar el aprendizaje de esta capacidad y el desarrollo de actividades formativas que ayuden a lograrlo.

La capacidad de evaluar críticamente la información es especialmente importante hoy en día y es una competencia informacional básica que recogen las más importantes normas bibliotecarias sobre alfabetización en información elaboradas por la ALA, SCONUL o ANZIIL. Estas normas o estándares se hacen eco de la necesidad, especialmente en la sociedad de la información y del conocimiento, de que la gente sea capaz de identificar sus necesidades de información, de saber buscarla, evaluarla, organizarla y utilizarla adecuadamente.

Quizá el precedente más cercano de la alfabetización informacional sea la formación de usuarios en las bibliotecas. Este servicio ha tenido como objetivo dar a conocer a los usuarios la institución, los servicios que presta, sus fondos y las distintas herramientas que pone a su disposición para buscar los documentos que necesite. Sin embargo, en la actualidad la gente dispone de más medios a su alcance para encontrar información y no cree que deba acudir a las bibliotecas para localizarla. Por tanto, la formación que necesita la gente va más allá del uso de la biblioteca y de sus fondos, debe ser capaz de encontrar, evaluar y usar la información eficazmente utilizando las múltiples fuentes y canales que existen. Como indica Barry (1999), la localización de información en una biblioteca era una tarea finita, dentro de los límites de los fondos existentes, y que consistía en la habilidad de utilizar los catálogos y los sistemas de clasificación de las estanterías; pero identificar y localizar recursos en un mundo electrónico puede que sea una tarea casi infinita. A medida que se pasa de una era tradicional a una electrónica, se intensifica la necesidad de habilidades de información.

Los bibliotecarios, como expertos en la búsqueda, selección y organización de la información, están en condiciones óptimas para la formación de estas competencias. Una importante labor que deben llevar a cabo, habida cuenta de la cada vez mayor desintermediación en el acceso a la información, es la capacitación de los usuarios en el uso de la 
información, que haga que el acceso autónomo a la misma no lleve a pérdidas de parte del conocimiento disponible, sino a un aprovechamiento satisfactorio (GÓMEZ; BENITO, 2001).

La evaluación de los recursos de información es una parte fundamental del proceso de selección de los fondos que integrarán la colección de la biblioteca. Las pautas que se han utilizado tradicionalmente para el formato impreso se han adaptado a los nuevos tipos y soportes documentales, primero a los CD-Roms y otros soportes ópticos, y luego a los documentos disponibles en internet. Esta adaptación no ha supuesto una ruptura total, hay criterios de evaluación que siguen vigentes, pero sí es cierto que se han incorporado otros criterios de evaluación nuevos y que la verificación de la calidad de los recursos es un poco más compleja, puesto que en internet, al no existir el filtro previo de una editorial, el usuario o el bibliotecario se convierte en el único filtro.

Los principales tipos de formación que ofrecen las bibliotecas sobre evaluación de la información son las guías o tutoriales y los cursos de formación, sean estos de carácter específico o formen parte de programas más amplios de alfabetización informacional.

\subsection{Tutoriales}

Existe una gran variedad de tutoriales o guías elaboradas por bibliotecas con el fin de ayudar a sus usuarios a valorar por sí mismos la calidad y la utilidad de la información que encuentran en internet (MANDALIOS, 2013). La mayoría de estas guías explican qué aspectos se deben tener en cuenta para determinar la calidad de los recursos, qué criterios se deben valorar y, en algunos casos, inluyen un cuestionario o checklist para facilitar la evaluación de los recursos. Algunos ejemplos de buenas prácticas serían los diseñados por la biblioteca de la Universidad Virginia Tech ${ }^{1}$ y por la Universidad Johns Hopkins ${ }^{2}$, que, además de recoger los principales criterios a tener en cuenta para evaluar un recurso de internet, incluyen una explicación de la importancia y utilidad de cada aspecto que debemos analizar y nos ofrece claves sobre dónde debemos fijarnos para encontrar la información necesaria para la evaluación; o el de la biblioteca de la Universidad de Albany ${ }^{3}$, que es interactivo y cuenta con actividades y preguntas que permiten a los usuarios comprobar sus conocimientos y sus habilidades.

A pesar de la utilidad de estos tutoriales o de los checklist para una primera toma de contacto con el tema, según diversos autores es necesaria la utilización de otras metodologías más flexibles para conseguir un aprendizaje en profunidad, ya que la evaluación crítica de la calidad y utilidad de los recursos de información es algo bastante complejo. Así lo constataron Calkins y Kelley (2007) con un grupo de estudiantes universitarios que, después de haber trabajado con guías de evaluación de recursos en internet, tenían dificultades para aplicarlas a casos reales.

\footnotetext{
${ }^{1}$ Disponible en: <http://www.lib.vt.edu/instruct/evaluate/index.html>. Consultado en: 16 Mayo 2013.

${ }^{2}$ Disponible en: <http://guides.library.jhu.edu/evaluatinginformation>. Consultado en: 16 Mayo 2013.

${ }^{3}$ Disponible en: <http://library.albany.edu/usered/webeval/>. Consultado en: 16 Mayo 2013.
} 
Para Meola (2004) el modelo basado en checklists es difícil de implementar en la práctica, dada la heterogeneidad de los recursos en la web, y adolece de un excesivo mecanicismo, que puede dar lugar a una infravaloración del pensamiento crítico y la reflexión. Este autor es más partidario de una aproximación contextual basada en "usar la información para evaluar información" y propone tomar como modelo y examinar los recursos que ya han sido evaluados por profesionales, la comparación de estos recursos con otros y la corroboración. También lo es Mandalios (2013), quién propone una herramienta denominada RADAR, basada en esos principios, que sirve de guía para comprender la evaluación de recursos en internet y profundizar en cuestiones como la relevancia, la autoridad o la motivación de los recursos.

\subsection{Cursos de formación}

Con respecto a los cursos de formación impartidos desde las bibliotecas, lo más habitual es que la enseñanza de evaluación de recursos de información en internet esté integrada en cursos más amplios de alfabetización informacional, aunque eso depende de cada caso y también pueden darse curso monográficos sobre ese tema en particular. Las bibliotecas llevan años implicadas en la enseñanza de competencias informacionales (al margen de la tradicional "formación de usuarios"), de hecho, el Euroreferencial en Información y Documentación (ECIA, 2004) contempla las acciones formativas como competencia fundamental de los profesionales de la documentación y ya se han incorporado a los planes docentes de las titulaciones de biblioteconomía y documentación asignaturas orientadas a que los titulados sean capaces de formar a los usuarios de las bibliotecas en este tipo de competencias.

En el ámbito universitario, la incorporación de asignaturas relacionadas con la búsqueda, evaluación y uso de la información en los planes de estudios de diversas titulaciones se ha dado desde hace tiempo, en especial en las universidades francesas, canadienses y anglosajonas y en menor medida en las españolas, siendo el efecto de tales cursos una mejora en el rendimiento de los alumnos (GÓMEZ, 2000). En otros ámbitos, como el de la biblioteca pública o escolar, la impartición de formación en competencias informacionales ha sido más costosa, quizá debido a que muchas de ellas cuenten con poco personal, tengan otras prioridades o que la formación no esté suficientemente recogida en las estructruas y planes de la organización. Sin embargo, si miramos en perspectiva su trayectoria, se puede observar que ha ido progresando paulatinamente y cada vez hay más iniciativas en este sentido (GÓMEZ, 2009). Como observan Gómez y Pasadas (2007) al analizar la situación internacional sobre la alfabetización informacional desde las bibliotecas públicas, se aprecia que hay un notorio reconocimiento por parte de la comunidad bibliotecaria de la necesidad de reorganizarse para dar mayor cabida a las actividades relacionadas con la formación en competencias informacionales. 


\section{Herramientas para el acceso a recursos de calidad}

Además de las acciones formativas orientadas a capacitar a los usuarios en evaluación de recursos de información en internet, las bibliotecas también pueden ayudarlos evaluando y seleccionando recursos de calidad que pueden ser de su interés, algo que los usuarios de las bibliotecas demandan desde hace tiempo (LUBANS, 1999). De esta forma podrán ahorrar tiempo en las búsquedas y en la evaluación o, al menos, disponer de una serie de recursos que saben que han pasado por un proceso de filtrado.

Las deficiencias de los buscadores (limitaciones es las búsquedas, falta de criterios de calidad en la selección de recursos y escasa descripción de los mismos) ofrecen argumentos de peso para diseñar mecanismos alternativos de organización de los recursos web y prefiguran la orientación y las principales características de los sistemas de acceso organizados desde las bibliotecas (ESTIVILL; ABADAL, 2000). La forma en que se organicen y se ofrezcan los recursos seleccionados a los usuarios puede variar en función del tipo de biblioteca, del tipo de usuarios o de los recursos con que cuente la biblioteca, y puede ser desde una mera lista de enlaces sobre un tema, a su integración en el catálogo, pasando por la creación de bases de datos o directorios de recursos.

\subsection{Listados de enlaces}

Los listados de enlaces son la forma más simple de identificar recursos de información relevantes sobre un tema y fueron la primera aproximación a la inclusión de recursos de internet en las bibliotecas. Según O'Leary (2000, p. 38), los bibliotecarios fueron de los primeros usuarios de la web y, siguiendo su instinto profesional, no tardaron en crear colecciones de enlaces que pudieran ser de utilidad para sus usuarios.

En un principio estos listados contenían enlaces ordenados alfabética o temáticamente o agrupados según su tipología, generalmente sin descripción formal ni de su contenido. Con el paso del tiempo, algunos de ellos fueron incluyendo algún elemento descriptivo que ayudara al usuario a conocer el posible interés del recurso, algo que hoy en día es más habitual. Según Estivill y Abadal (2000), aunque tienen cierta utilidad, es una aproximación demasiado elemental, que no tiene en cuenta las posibilidades tecnológicas existentes, adolece de falta de normalización en las descripciones y sólo permite una búsqueda lineal, sin explotar ningún mecanismo de recuperación de información.

\subsection{Guías temáticas}

Las guías temáticas son listas de recursos creadas para ayudar a los estudiantes en sus necesidades de investigación (STALEY, 2007). En un principio, antes de la aparición de internet, se limitaban a los recursos de 
que disponía la biblioteca. De ahí que una de las primeras definiciones de guía temática fue "un tipo de mapa de recursos de la biblioteca, que sirve de localizador para el usuario de la biblioteca que está comenzando a buscar información sobre un tema" (STEVENS; CANFIELD; GARDNER, 1973). Hoy en día estas guías recogen todo tipo de recursos, tanto los propios de la biblioteca como otros que pueden consultarse en internet, lo que supone una de las principales diferencias con respecto a los listados de recursos.

La forma que adoptan estas guías es generalmente la de una página web o la de un documento en pdf, aunque también es posible utilizar las tecnologías de la Web 2.0, como pueden ser las wikis o el uso de Delicious (WAKEHAM et al., 2012; MORRIS; DEL BOSQUE, 2010), o su adaptación a dispositivos móviles 0 a formatos audiovisuales (STRUTIN, 2008). También existe software específico, como Libguides ${ }^{4}$, que son sistemas de gestión de contenidos, que permiten la creación y personalización de guías temáticas en un entorno web.

Su estructura y tamaño varía de unas bibliotecas a otras, pero en general no pretenden ser exhaustivas, sino ofrecer una cuidada selección de recursos de utilidad sobre un tema o una asignatura en concreto. De hecho, según Dahl (2001), estas guías son menos útiles cuando tratan de abarcar temas muy amplios y son especialmente apropiadas para temas más específicos, como por ejemplo una asignatura (COURTOIS; HIGGINS; KAPUR, 2003). Por lo general cuentan con enlaces a recursos de internet, obras de referencia, manuales, catálogos y bases de datos, diccionarios y glosarios, congresos o eventos relacionados con la materia y otro tipo de fuentes, en función de la disciplina y de la audiencia a la que van dirigidas, y suelen ir acompañadas de una breve descripción del contenido de dichos recursos.

La utilidad de estas guías radica sobre todo en el hecho de contar con un primer punto de partida a la hora de obtener información sobre un tema. Su uso es considerable y la valoración por parte de los usuarios es en general positiva (COURTOIS; HIGGINS; KAPUR, 2003; WAKEHAM et al., 2012). Además tiene un efecto positivo en las personas que las usan, ya que, como observó Galvin (2005), los estudiantes que hacen uso de las guías temáticas tienden a utilizar más recursos académicos.

No obstante, también se han encontrado puntos de mejora de las guías temáticas y numerosos autores abogan por explotar al máximo las posibilidades tecnológicas disponibles, en especial la Web 2.0 (VILENO, 2007; WAKEHAM et al., 2012; MORRIS; DEL BOSQUE, 2010), por tener más en cuenta las opiniones y el feedback de los usuarios (VILENO, 2007; JACKSON; PELLACK, 2004) y por intentar, en la medida de lo posible, personalizar este tipo de herramientas para ajustarlas mejor a las necesidades de los distintos tipos de usuarios (OUELLETTE, 2011; REEB; GIBBONS, 2004).

4 Disponible en: <http://www.springshare.com/libguides/>. Consultado en: 16 Mayo 2013. 


\subsection{Bases de datos y directorios}

La creación de bases de datos y directorios de recursos de internet, también llamados directorios analíticos, pasarelas temáticas o subject gateways, supuso el primer paso hacia su gestión bibliotecaria, ya que es ahora cuando estos reciben un tratamiento técnico más allá de su mera selección o breve descripción (BURNETT; SEURING, 2001). Su inclusión en una base de datos o en un directorio analítico presupone una mayor consideración de estos recursos y significa una descripción en mayor profundidad, que recoja la información descriptiva en una serie de campos, más posibilidades de búsqueda $y$, en general, un volumen considerable de recursos, que justifica dicho tratamiento técnico.

La forma de acceso a los registros suele ser a través de una página web propia, en la que el usuario puede navegar por un estructura clasificatoria, generalmente temática, aunque puede haber otras, y en algunos casos puede utilizar un buscador interno, más o menos avanzado. En función del grado de desarrollo de la herramienta, los registros pueden ir desde una ficha compuesta por el título del recurso, el enlace al recurso y una breve descripción del contenido, hasta un registro completo con una serie de campos (autor, título, resumen, descriptores...) que suelen ajustarse a los campos del formato Dublin Core, del formato MARC o al de otro formato normalizado de descripción bibliográfica. Además, es aconsejable que estas herramientas cuenten con una política de selección de recursos explícita, que permita a los usuarios conocer qué criterios se han utilizado para la inclusión de recursos y, por tanto, saber cómo se han filtrado.

Algunas de las ventajas que supone este modelo son la facilidad de uso por parte de los usuarios, que pueden buscar la información de forma intuitiva a través de un formulario de búsqueda o navegando por su estructura clasificatoria; la posibilidad de intercambiar registros y de trabajar colaborativamente al utilizar una descripción normalizada; su carácter gratuito, que permite a personas ajenas a la biblioteca poder utilizar estas herramientas; y su rigor, ya que la selección generalmente es llevada a cabo de forma conjunta por expertos en el tema y por bibliotecarios. Además estas bases de datos y directorios pueden utilizarse como base para elaborar guías temáticas, permitiendo así que un mismo recurso pueda estar en varias guías (VILENO, 2007).

\subsection{Integración en el catálogo}

Aunque tradicionalmente en el catálogo se incluían únicamente los registros de los fondos que eran propiedad de la biblioteca, a medida que los recursos electrónicos dejaron de estar físicamente en sus edificios y se accedía a ellos a través de la red se fueron incorporando al catálogo, principalmente las revistas electrónicas y las bases de datos. La inclusión también de los recursos gratuitos disponibles en internet es una opción que ha sido contemplada casi desde el momento en que estos recursos 
aparecieron (WOODWARD, 1996). Sin embargo, debido a las características de estos recursos y de los propios catálogos, no hay unanimidad acerca de si esta es o no la mejor opción.

Algunos de los principales inconvenientes han sido recogidos por Lam (2000), quien, al analizar los esfuerzos en la década de los 90 por integrar recursos de internet en los catálogos de las bibliotecas, destaca que su naturaleza caótica e inestable, así como los cambios que supone su actualización, suponen un importante inconveniente que hace que muchas bibliotecas prefieran no incluirlos en los catálogos y se decanten por otras opciones. Otro de los problemas es que, debido al elevado volumen de recursos de calidad disponibles en internet, una catalogación completa como la que se hace con los libros, aunque es posible ya que el formato MARC lo permite a través de la etiqueta 856 , podría ser muy costosa y se necesitaría mucho tiempo para llevarla a cabo (GORMAN, 1999).

A pesar de estos inconvenientes, muchos autores consideran que merece la pena el esfuerzo, puesto que estos recursos pueden ser muy valiosos y merecen un tratamiento similar al del resto de los fondos. Las principales razones esgrimidas son que se puede optar por registros en formato MARC con menos profundidad o utilizar otros formatos mejor adaptados a los recursos electrónicos, como el Dublin Core o el RDA y que de esta forma se permite un único punto de acceso, que evite a los usuarios tener que buscar la información en diferentes fuentes (ESTIVILL; ABADAL, 2000; BROWN; MEAGHER, 2008). Además, esta opción no es incompatible con el acceso a través de directorios o guías temáticas (BURNETT; SEURING, 2001). Este enfoque mixto, que contempla la incorporación al catálogo mediante registros en formato MARC y el aprovechamiento de esos registros para crear guías o directorios, es una opción que ha tenido bastante aceptación en la comunidad bibliotecaria (BOYDSTON; LEYSEN, 2002).

\section{Conclusiones}

La sobreabundancia de información que ha supuesto la aparición de internet no ha traído aparejada mecanismos de control y organización que permitan una eficaz búsqueda y recuperación de información de calidad. A pesar de la potencia cada vez mayor de los buscadores, los problemas para identificar recursos de calidad siguen existiendo en un entorno en el que conviven documentos de gran valor $y$ otros completamente irrelevantes.

La labor que pueden y deben cumplir las bibliotecas en este contexto es fundamental, ya que, al ser su función por excelencia ser intermediarios entre la información y los usuarios, cuentan con la experiencia y el bagaje de conocimientos necesarios para poder ayudar a la gente a localizar información de calidad independientemente de su soporte o formato. Lejos de perder peso en la sociedad del conocimiento, las bibliotecas tienen más importancia que nunca, ya que nunca antes 
había existido tanta información y para poder ser recuperada eficazmente ésta debe ser seleccionada, analizada y organizada adecuadamente.

En este trabajo se han descrito dos de las principales aproximaciones al papel de las bibliotecas en el acceso a recursos de calidad en la web: por un lado la inclusión de estos recursos en los fondos de la biblioteca mediante su identificación, selección, descripción y organización y, por otro, la formación para que los usuarios sean capaces por sí mismos de buscar, evaluar y utilizar la información disponible en internet de una forma eficaz.

\section{Referencias}

BARRY, C. A. Las habilidades de información en un mundo electrónico: la formación investigadora de los estudiantes de doctorado. Anales de Documentación, v. 2, p. 237-258, 1999.

BOYDSTON, J. M. K.; LEYSEN, J. M. Internet resources cataloging in ARL libraries. The Serials Librarian, v. 41, n. 3-4, p. 127-145, 2002.

BREIVIK, P. S. Student learning in the information age. Phoenix, AZ: The Oryx Press, 1998.

BROWN, C. C.; MEAGHER, E. S. Cataloging free e-resources: is it worth the investment? Interlending \& Document Supply, v. 36, n. 3, p. 135-141, 2008.

BURNETT, P.; SEURING, C. Organising access to free Internet resources: an overview of selection and management issues in large academic and national libraries with a view to defining a policy at Oxford University. Program: Electronic Library and Information Systems, v. 35, n. 1, p. 15$31,2001$.

CALKINS, S.; KELLEY, M. Evaluating Internet and scholarly sources across two disciplines: two case studies. College Teaching, v. 55, n. 4, p. 151156, 2007.

CODINA, L. Evaluación de recursos digitales en línea: conceptos, indicadores y métodos. Revista Española de Documentación Científica, v. 23, n. 1, p. 7-43, 2000.

CODINA, L.; ABADAL, E. Crítica del mundo digital: propuesta de categorización. Notas ThinkEPI, 2013. Disponible en: <http://www.thinkepi.net/critica-del-mundo-digital-propuesta-decategorizacion>. Consultado en: 16 Mayo 2013.

COURTOIS, M. P.; HIGGINS, M. E.; KAPUR, A. Was this guide helpful? Users' perceptions of subject guides. Reference Services Review, v. 33, n. 2, p. 188-196, 2003.

CROSS, D. D. Determining standards for sources of free information on the internet for inclusion in Academic Library Holdings by 2010. 2002. Disponible en: <http://dc.etsu.edu/etd/715/>. Consultado en: 16 Mayo 2013. 
DAHL, C. Electronic pathfinders in academic libraries: an analysis of their content and form. College \& Research Libraries, v. 62, n. 3, p. 227-237, 2001.

ECIA. Euroreferencial en Información y Documentación. Competencias y actitudes de los profesionales europeos de información y documentación. Madrid: $\quad$ SEDIC, $2004 . \quad$ v. $1 . \quad$ Disponible en: <http://www.certidoc.net/es1/euref1-espanol.pdf>. Consultado en: 16 Mayo 2013.

ESTIVILL, A.; ABADAL, E. Acceso a los recursos web gratuitos desde las bibliotecas. El Profesional de la Información, v. 9, n. 11, p. 4-20, 2000.

GALVIN, J. Alternative strategies for promoting information literacy. Journal of Academic Librarianship, v. 31, n. 4, p. 352-357, 2005.

GÓMEZ, J. A.; BENITO, F. De la formación de usuarios a la alfabetización internacional: propuestas para enseñar las habilidades de información. Scire: Representación y Organización del Conocimiento, v. 7, n. 2, p. 5383, 2001.

GÓMEZ, J. A. La alfabetización informacional y la biblioteca universitaria: organización de programas para enseñar el uso de la información. In: GÓMEZ, J. A. (Ed.) Estrategias y modelos para enseñar a usar la información: guía para docentes, bibliotecarios y archiveros. Murcia: KR, 2000. p. $169-255$.

GÓMEZ, J. A. Aprender a enseñar competencias informacionales a los usuarios: avances en la formación profesional en España. Anuario ThinkEPI, v. 3, p. 106-113, 2009.

GÓMEZ, J. A.; PASADAS, C. La alfabetización informacional en bibliotecas públicas: situación actual y propuestas para una agenda de desarrollo. Information Research, v. 12, n. 3, 2007. Disponible en: <http://informationr.net/ir/12-3/paper316.html>. Consultado en: 16 Mayo 2013.

GORMAN, M. Metadata or cataloguing? A false choice. Journal of Internet Cataloging, v. 2, n. 1, p. 5-22, 1999.

HERRING, J. E. Web site evaluation: a key role for the school librarian. School Library Monthly, v. 27, n. 8, p. 22-23, 2011.

JACKSON, R.; PELLACK, L. J. Internet subject guides in academic libraries: an analysis of contents, practices, and opinions. Reference \& User Services Quarterly, p. 319-327, 2004.

$\mathrm{KOHL}, \mathrm{D}$. F. Knowledge life cycles: renewal and obsolescence. El Profesional de la Informacion, v. 18, n. 4, p. 374-381, 2009.

LAM, V. T. Cataloging internet resources: why, what, how. Cataloging \& Classification Quarterly, v. 29, n. 3, p. 49-61, 2000. 
LUBANS JR, J. When students hit the surf: what kids really do on the internet. and what they want from librarians. School Library Journal, v. 45, n. 9, p. 144-47, 1999.

LUCASSEN, T.; SCHRAAGEN, J. M. Factual accuracy and trust in information: the role of expertise. Journal of the American Society for Information Science and Technology, v. 62, n. 7, p. 1232-1242, 2011.

MANDALIOS, J. RADAR: an approach for helping students evaluate Internet sources. Journal of Information Science, v. 39 , n. 4, p. 470-478, 2013.

MARTÍNEZ, F. J.; RODRÍGUEZ, J. V. Síntesis y crítica de las evaluaciones de la efectividad de los motores de búsqueda en la Web. Information Research, v. 8, n. 2, 2003. Disponible en: <http://informationr.net/ir/82/paper148.html>. Consultado en: 16 Mayo 2013.

MEOLA, M. Chucking the checklist: a contextual approach to teaching undergraduates web-site evaluation. Portal: Libraries \& the Academy, v. 4, n. 3, p. 331-344, 2004.

MORRIS, S. E.; DEL BOSQUE, D. Forgotten resources: subject guides in the era of Web 2.0. Technical Services Quarterly, v. 27, n. 2, p. 178-193, 2010.

O'LEARY, M. Grading the library portals. Online, v. 24, n. 6, p. 38-44, 2000.

OPPENHEIM, C. et al. The evaluation of WWW search engines. Journal of Documentation, v. 56, n. 2, p. 190-211, 2000.

OUELLETTE, D. Subject guides in academic libraries: a user-centred study of uses and perceptions. Canadian Journal of Information and Library Science, v. 35, n. 4, p. 226-241, 2011.

PINGDOM. Internet 2012 in numbers. 2013. Disponible en: http://royal.pingdom.com/2013/01/16/internet-2012-in-numbers/>. Consultado en: 16 Mayo 2013.

REEB, B.; GIBBONS, S. Students, librarians, and subject guides: improving a poor rate of return. Portal: Libraries and the Academy, v. 4, n. 1 , p. $123-130,2004$.

STALEY, S. M. Academic subject guides: a case study of use at San Jose State University. College \& Research Libraries, v. 68, n. 2, p. 119-140, 2007.

STEVENS, C.; CANFIELD, M.; GARDNER, J. Library pathfinders: a new possibility for cooperative reference service. College \& Research Libraries, v. 34 , n. 1 , p. 40-46, 1973.

STRUTIN, M. Making research guides more useful and more well used. Issues in Science \& Technology Librarianship, n. 55, 2008. Disponible en: <http://www.istl.org/08-fall/article5.html>. Consultado en: 16 Mayo 2013. 
VILENO, L. From paper to electronic, the evolution of pathfinders: A review of the literature. Reference Services Review, v. 35, n. 3, p. 434451, 2007.

WAKEHAM, M. et al. Library subject guides: a case study of evidenceinformed library development. Journal of Librarianship and Information Science, v. 44, n. 3, p. 199-207, 2012.

WOODWARD, J. Cataloging and classifying information resources on the Internet. Annual Review of Information Science and Technology, v. 31, p. 189-220, 1996. 